\title{
A Comparative Study between Opportunistic Lumbar Spine CT Scan and DEXA Scan in the Assessment of Osteoporosis
}

Kirzner $\mathbf{N}^{1 *}$, Gallagher $\mathbf{C}^{2}$, Kimmel $\mathrm{L}^{2}$, Fischer $\mathrm{D}^{3}$, Mc Laurin $\mathbf{W}^{3}$, Liew $\mathrm{S}^{3}$ and Humadi $\mathrm{A}^{1}$

${ }^{1}$ Department of Orthopaedics, Alfred Hospital, Prahran, VIC, Australia

${ }^{2}$ Department of Physiotherapy, Alfred Hospital, Prahran, VIC, Australia

${ }^{3}$ Department of Radiology, Alfred Hospital, Prahran, VIC, Australia

\section{Abstract}

Background: This retrospective study aimed to investigate a correlation between computed tomography (CT) imaging obtained opportunistically during screening for lumbar spine fractures and dual-energy x-ray absorptiometry (DEXA) scans.

Methods: A total of 159 patients over a 5-year study period who had undergone CT scan to investigate for a lumbar spine fracture and a DEXA scan within 12-months of each other were included in the study. Measurements of the region of interest (ROI) through the first 4 lumbar vertebrae were performed to establish Hounsfield unit (HU) values. Measurements for each level were made at three separate locations: mid-vertebral body, and just above and below the inferior and superior endplates, respectively. The HU values were correlated with T-scores obtained from DEXA scans and were further analyzed according to age and gender.

Results: There was a statistically significant correlation between HU values with T-score. Using the Pearson correlation coefficients, a moderate correlation of $\mathrm{HU}$ value to hip DEXA for T-score. There was a moderate-strong correlation between DEXA lumbar spine with mid-vertebral body $\mathrm{HU}$, with $\mathrm{L} 3$ having the strongest correlation $\left(r^{2}=0.7269\right)$. The normal group had a mean $\mathrm{HU}$ value of $139.3(95 \% \mathrm{Cl} 119-159.2)$, the osteopenic group had a mean of 105.9 (95\% Cl 90.4-123.4), and the osteoporotic group had a mean of 72.4 (95\% Cl 60.1-81.7).

Conclusion: This current study demonstrated that opportunistic CT imaging can be utilized to infer bone quality and provide information about the presence of osteoporosis and subsequently fracture risk without the need for additional imaging, radiation exposure, cost, or patient time.

Keywords: Osteoporosis; Computed Tomography (CT); DualEnergy X-ray Absorptiometry (DEXA); Opportunistic screening

\section{Introduction}

Osteoporosis is a growing public health concern, especially with an ageing population as the proportion of patients over the age of 65 years continues to rise. The annual incidence in Australia is approaching a million people (3.8\%) and is estimated to rise to 1.5 million by 2022 [1]. Osteoporosis is characterized by an age-related reduction in bone strength that predisposes affected individuals to low-energy fragility fractures. These can result in chronic pain, disability and loss of independence, which are associated with substantial health care costs approaching \$1 billion (Australian dollars) annually [1]. Despite this burden, osteoporosis remains substantially underdiagnosed and subsequently undertreated. Nearly one-half of women indicated for screening do not undergo testing, and certain high-risk populations have screening rates less than $10 \%[2,3]$. Therefore, safe and costeffective methods to increase detection of this condition are needed.

Dual-energy x-ray absorptiometry (DEXA) of the hips and lumbar spine has traditionally been the gold standard for quantifying bone mineral density (BMD) and remains the diagnostic tool preferred by the International Society for Clinical Densitometry (ISCD) [4]. Whilst DEXA has been correlated with fracture risk and treatment efficacy [4], variability in body composition can lead to errors of up to $20 \%[5,6]$. Furthermore, DEXA cannot be used in patients with spinal deformities such as scoliosis and degenerative changes as osteophytes artificially elevate DEXA results [7]. These deficiencies and the low rates of DEXA screening, make utilizing readily available patient data to infer bone quality an appealing and convenient solution.

Measuring BMD by using quantitative computed tomography (CT) was first described by Genant and Boyd in the 1970's [8]. However, despite its early inception and accuracy, quantitative CT was confined to research purposes due to the high doses of ionizing radiation and relatively long scanning times required [9]. The widespread availability and use of CT for other indications and advancements in CT technology [10] have led to resurgence in the idea of opportunistic CT imaging for osteoporosis screening. Recent studies have suggested that the use of Hounsfield units (HUs) from CT scanning may be a useful surrogate marker for estimating BMD of the spine [11-14].

The purpose of this study was to determine if there is a correlation between DEXA-based bone density assessment and diagnostic CT that was opportunistically obtained during investigations for lumbar spine fractures. Currently there is a paucity of evidence on opportunistic CT that limits its broader applicability and use. We hypothesized that opportunistic CT scans can be a reliable method to screen for patients with bone mineral disease and subsequently evaluate their risks of fractures.

\section{Materials and Methods}

\section{Patients}

Using our hospital's electronic database and the orthopaedic unit audit, we identified all patients who had undergone a CT lumbar spine

*Corresponding author: Nathan Kirzner, Registrar, Department of Orthopaedics, Alfred Hospital, Prahran, VIC, Australia, Tel: +0401 599 622; E-mail: Nathan. kirzner@gmail.com

Received February 09, 2019; Accepted February 19, 2019; Published February 25, 2019

Citation: Kirzner N, Gallagher C, Kimmel L, Fischer D, Mc Laurin W, et al. (2019) A Comparative Study between opportunistic lumbar spine CT scan and DEXA Scan in the Assessment of Osteoporosis. J Spine 8: 434. doi: 10.4172/2165-7939.1000434

Copyright: @ 2019 Kirzner N, et al. This is an open-access article distributed under the terms of the Creative Commons Attribution License, which permits unrestricted use, distribution, and reproduction in any medium, provided the original author and source are credited. 
at our level 1 trauma centre between 1 January 2012 and 1 January 2017. The Alfred hospital's human research ethics committee provided ethical approval for the study.

Our inclusion criteria were patients over 30 years old with CT lumbar Spine and DEXA scan within 12 months of each other. Patients were excluded from the study if they had lumbar spine instrumentation, previous vertebroplasty or spinal fusion, invalid DEXA scan results due to degeneration, fracture, or deformity, or incomplete DEXA. 159 patients from around 2000 patients reviewed, were enrolled in the study. Of these 66 were men and 95 women with a mean age of 65.7 years (32 to 91). 24 of these patients had lumbar spine fractures detected on CT scan.

T-scores for the first 4 lumbar vertebrae and hips were retrieved from DEXA scans that had previously been performed. A $0.5 \mathrm{~cm}$ multislice CT scanner (Aquilion Precision; Canon, USA) was utilized for all patients. Using standard picture archiving and communication system (PACS) software, measurements of an elliptical region of interest (ROI) through the first 4 lumbar vertebrae was performed to establish Hounsfield unit (HU) values. The HU measurement for each vertebra was obtained by using a protocol described by Schreiber et al. (12). Measurements for each level were made at three separate locations: mid-vertebral body, and just above and below the inferior and superior endplates, respectively (Figure 1). Hounsfield unit measurements were obtained by two blinded independent observers including a radiologist and an orthopaedic surgeon and the average was taken.

\section{Statistical analysis}

One-way analysis of variance (ANOVA) was used to measure the differences in $\mathrm{HU}$ values between age groups and gender. T-tests were used for post hoc analysis with a p-value $<0.05$ considered statistically significant. Pearson correlation coefficients were used to determine connections between $\mathrm{HU}$ value and DEXA T-scores.

\section{Results}

A significant correlation was observed between age and $\mathrm{HU}$ $(p<0.05)$ when the 159 patients were analyzed according to decade of life. A steady decline in $\mathrm{HU}$ values with increasing age was also noted, ranging from a mean of $151.4 \mathrm{HU}$ in the 3rd decade of life to 91.2 HU in the 9th decade of life (Figure 2). A Subgroup analysis was also performed between mean $\mathrm{HU}$ and gender. The mean $\mathrm{HU}$ in females ranged from 160 in young adults to less than 84 in those in their nineties, with a predictable decline in $\mathrm{HU}$ at the time of menopause. The male data showed a more gradual decrease in $\mathrm{HU}$ with age, ranging from 165.3 in young adults to just under 100 in those in their eighties.
The T-scores of both hip and lumbar spine were obtained for the 159 patients who underwent DEXA scans within one year of their CT imaging. T-scores ranged from -4.1 to +2.5 (mean $-1.1+/-0.89)$ for the lumbar spine and -4.4 to $+1.5(-1.3+/-1.17)$ for the hip. Each vertebral level was analyzed separately. Using the Pearson correlation coefficients, a moderate correlation of HU value to hip DEXA for T-score (Table 1). There was a moderate-strong correlation between DEXA lumbar spine with mid-vertebral body HU, with L3 having the strongest correlation $\left(\mathrm{r}^{2}=0.7269\right)$. All obtained correlations were significant $(\mathrm{p}<0.001)$.

Based on the World Health Organization's guideline [15], the 159 patients' lumbar vertebrae and hip T-scores were stratified into three groups : normal (-1.0 or greater), osteopaenic (less than -1.0 and greater than -2.5$)$, and osteoporotic (-2.5 or less) (Figure 2). The mean HU values were 139.3 (95\% CI 119-159.2) for the normal group, 105.9 (95\% CI 90.4-123.4) for the osteopenia group, and 72.4 (95\% CI 60.181.7 ) for the osteoporotic group (Figure 3). The differences in mean $\mathrm{HU}$ values between groups were all significant $(\mathrm{p}<0.001)$.

In the 24 patients with lumbar spine fractures HU measurements at the fractured vertebral body were significantly lower in the fracture group compared with matched control (90.6 vs 121.3; $\mathrm{p}=0.007$ ). Comparing Global HU measurements between the fracture and no fracture groups throughout the lumbar spine demonstrated significantly lower HU measurements in the fracture group (106.7 vs 125.29; $\mathrm{p}<0.05$ ). When reviewing the DEXA scores of these patients that had fractures, the mean DEXA T-scores were between the normal and osteopaenic range.

\section{Discussion}

This current study demonstrated that opportunistic CT imaging can be utilized to infer bone quality and provide information about the presence of osteoporosis and subsequently fracture risk. Furthermore, this can be done at no additional cost or radiation exposure and with minimal effort. The method that we used requires a negligible amount of training and time, and can be performed either prospectively by the interpreting radiologist or retrospectively by a radiologist or nonradiology trained clinician with comparable accuracy.

DEXA based T-scores, defined by the number of standard deviations below the mean peak bone mass of a young and healthy adult, have been the gold standard for quantifying BMD. However, osteoporosis screening with DEXA remains underutilized potentially resulting in fragility fractures, which lead to chronic pain and disability and cost the healthcare system considerable amounts of money to treat. Therefore, in recent times there has been a growing interest in the opportunistic use of CT imaging to screen for osteoporosis.

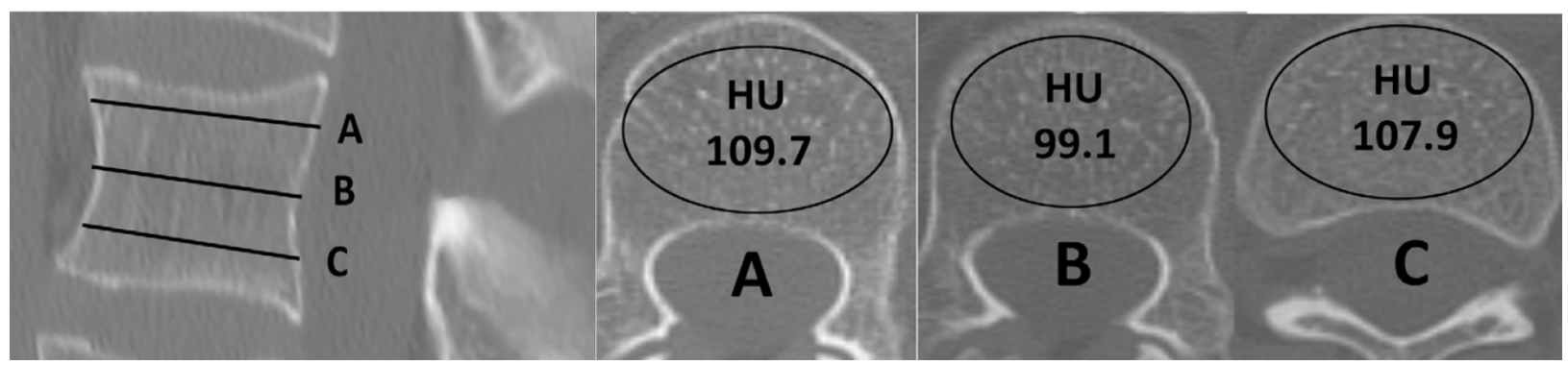

Figure 1: Computed tomography (CT) scans of lumbar vertebra L3 illustrating the method of determining Hounsfield unit (HU) values by using a picture archiving and communication system (PACS). From a reconstructed sagittal image, we select three axial planes of interest: slice (A) is taken just inferior to the superior endplate, slice $(B)$ is from the middle of the vertebral body, and slice $(C)$ is taken just superior to the inferior endplate. The PACS program automatically calculates the mean $\mathrm{HU}$ value of the regions of interest which are marked with ellipses in the figure. The average of $\mathrm{HU}$ values from three axial cuts, which is $89 \mathrm{HU}$ in this case, was used for the analysis. 


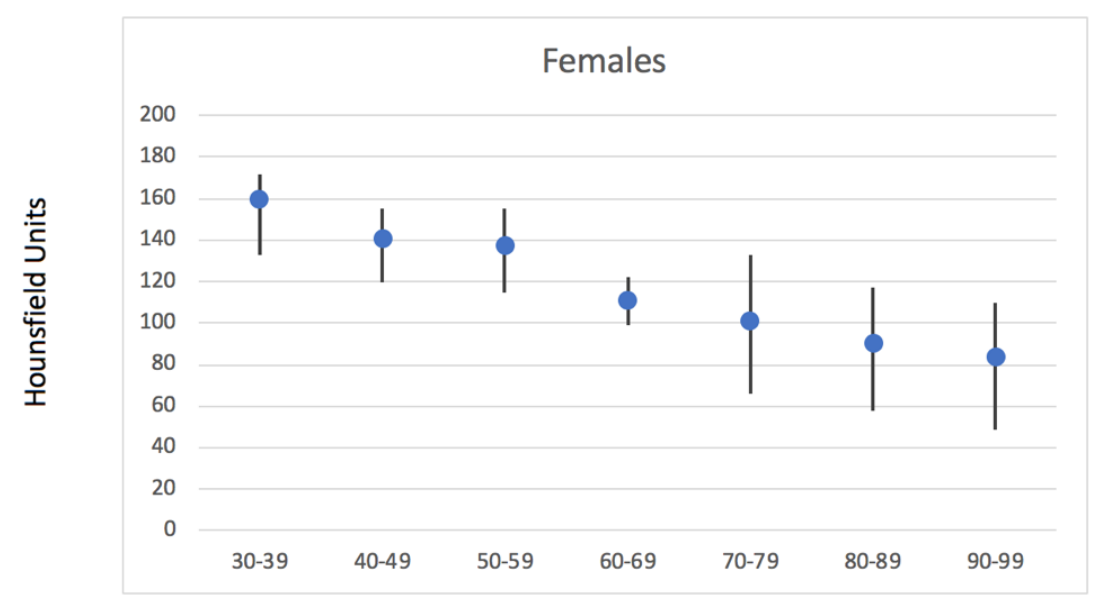

Age range (Yrs)

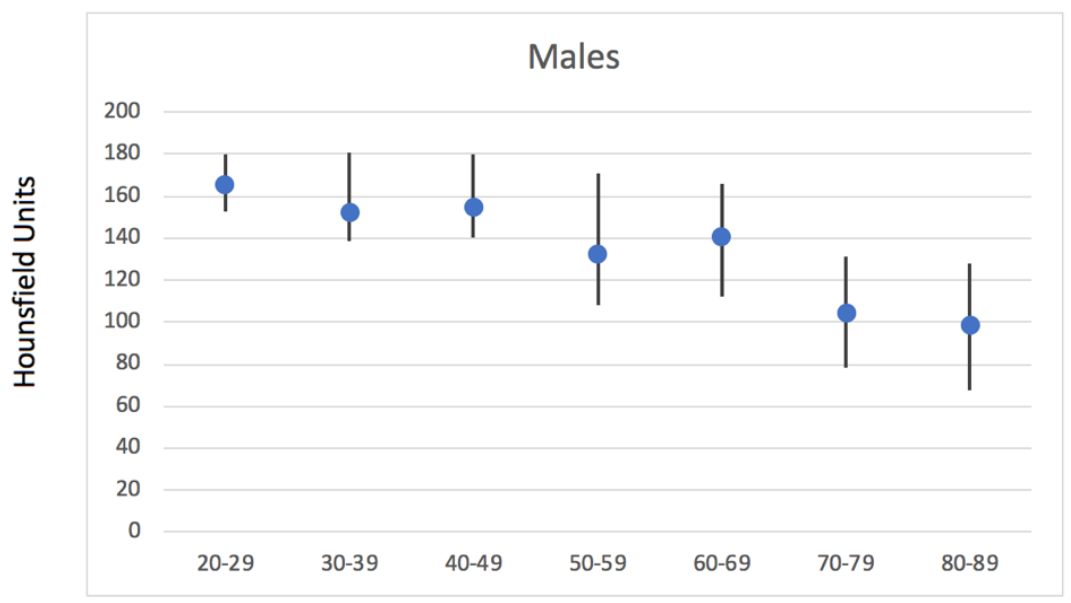

Age range (Yrs)

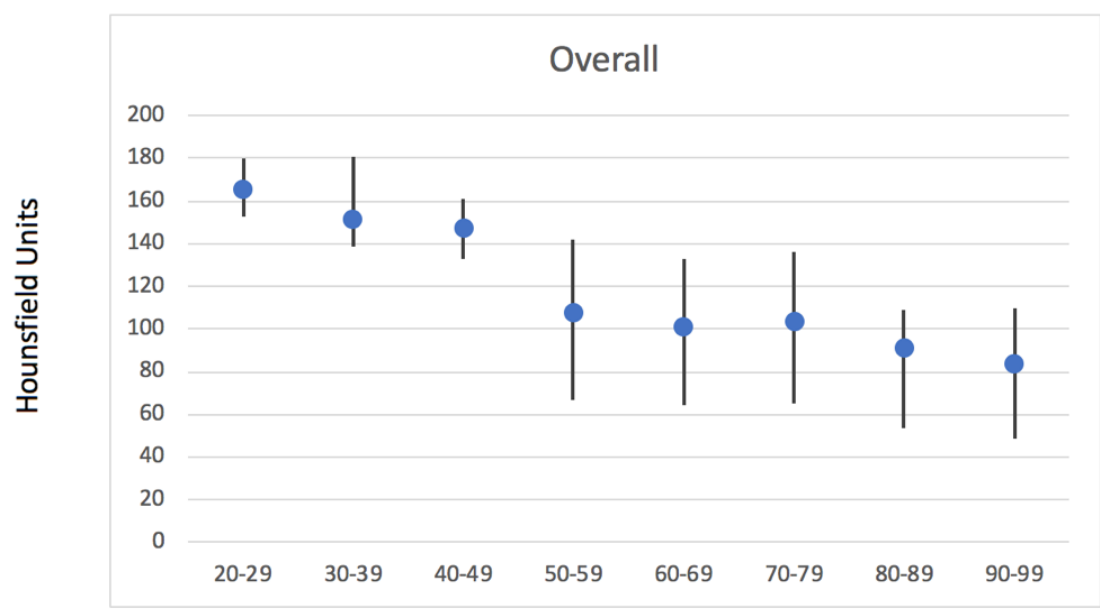

Age range (Yrs)

Figure 2: Normative data of HU values in females (A), males (B), and overall (C) stratified by decade of life. 
Lee and co-authors in a study 128 females undergoing lumbar CT for back pain found a significant positive correlations $(p<0.001)$ of $\mathrm{HU}$ value with bone mineral density and T-scores [9]. They demonstrated that subjects with a normal bone density had a mean lumbar $\mathrm{HU}$ value of 120.8 , those with osteopenia had a mean lumbar HU value of 78.8 , and those with osteoporosis had a mean lumbar HU value of 54.7. Another study by Schreiber co-authors of 80 consecutive presenting trauma patients also found a good correlation between lumbar HU value to DEXA for both BMD and T-score [16]. In this case they established the threshold for diagnosing osteoporosis based on lumbar spine HU measurements to be 78.5 (+/- 32.4). In another study utilizing abdominal CT scans performed for variation indications, Pickhardt coauthors found that an L1 HU threshold of $110 \mathrm{HU}$ was more than $90 \%$ specific for distinguishing osteoporosis, while positive predictive values for osteoporosis were $68 \%$ or greater at thresholds less than $100 \mathrm{HU}$ [17]. Our present study also found $\mathrm{HU}$ values obtained from CT scans to be significantly correlated to T-score. Our results were comparable to the above studies with the normal and osteopaenic groups having mean $\mathrm{HU}$ values greater than 100 , whilst the osteoporotic group had a mean of $72.4 \mathrm{HU}$.

Identifying persons with very low BMD by CT (for example, $<100$ $\mathrm{HU}$ ) might allow for rapid identification of high-risk cohorts in whom further evaluation or treatment is warranted. Conversely, patients with $>150 \mathrm{HU}$ on CT may be considered to have a normal BMD, effectively ruling out osteoporosis, making further investigation with a DEXA

\begin{tabular}{|c|c|c|}
\hline Vertebral level & DXA Hip vs. HU mid VB & $\begin{array}{c}\text { DXA Spine vs. HU } \\
\text { mid VB }\end{array}$ \\
\hline L1 & 0.5849 & 0.6723 \\
\hline L2 & 0.5037 & 0.5888 \\
\hline L3 & 0.6349 & 0.7269 \\
\hline L4 & 0.6862 & 0.6425 \\
\hline
\end{tabular}

Table 1: Pearson correlations between DXA of hip and spine versus Hounsfield unit (HU) at the mid vertebral body (VB) level of lumbar spines 1-4. scan unnecessary. Whilst CT scanning at this stage should not be used for primary screening purposes or as a substitute for DEXA, when the data is already available it can be useful to identify at-risk patients. In the setting of trauma, this information can provide insight into injury aetiology and offer the treating surgeon immediate knowledge of the bone quality. This may not only affect the surgical decision making, but enable the surgeon to initiate treatment or to refer the patient for appropriate further management.

In degenerative spine disease and cases of deformity requiring instrumentation, the ability to identify the presence of osteoporosis is critical as its presence will affect the capacity to achieve stability. It is known that the presence of spondylophytes, one component of degenerative spine disease, impairs DEXA-BMD measurements in the spine [18-20]. In these patients BMD is systematically overestimated and DEXA scans are unreliable. In these cases CT imaging, which will already be obtained for preoperative planning, could be utilized to establish the presence of osteoporosis. CT has the added advantage of being able to accurately identify unsuspected osteoporotic compression fractures, which are diagnostic of osteoporosis regardless of the patient's DEXA T-score [21]. Several studies including our current study have shown normal and osteopaenic DEXA T-scores to be prevalent among patients with vertebral fractures [17,22]. Again this finding highlights the limitations of DEXA, particularly in terms of BMD overestimation related to degenerative changes $[21,23]$.

This study has a number of limitations. Firstly, the data of our study came from one CT scanner and variability among different machines may limit the broad applicability of this technique. Despite this, our HU thresholds were comparable to previous studies. Another limitation is the maximum one year interval between CT and DEXA may have influenced the results. However, this seems unlikely unless the patients were undergoing osteoporosis treatment at the time. This study is also limited by its retrospective nature and the possibility that bias occurred. Despite this potential drawback, its impact was nullified as all the data was obtained by two independent, blinded clinicians.

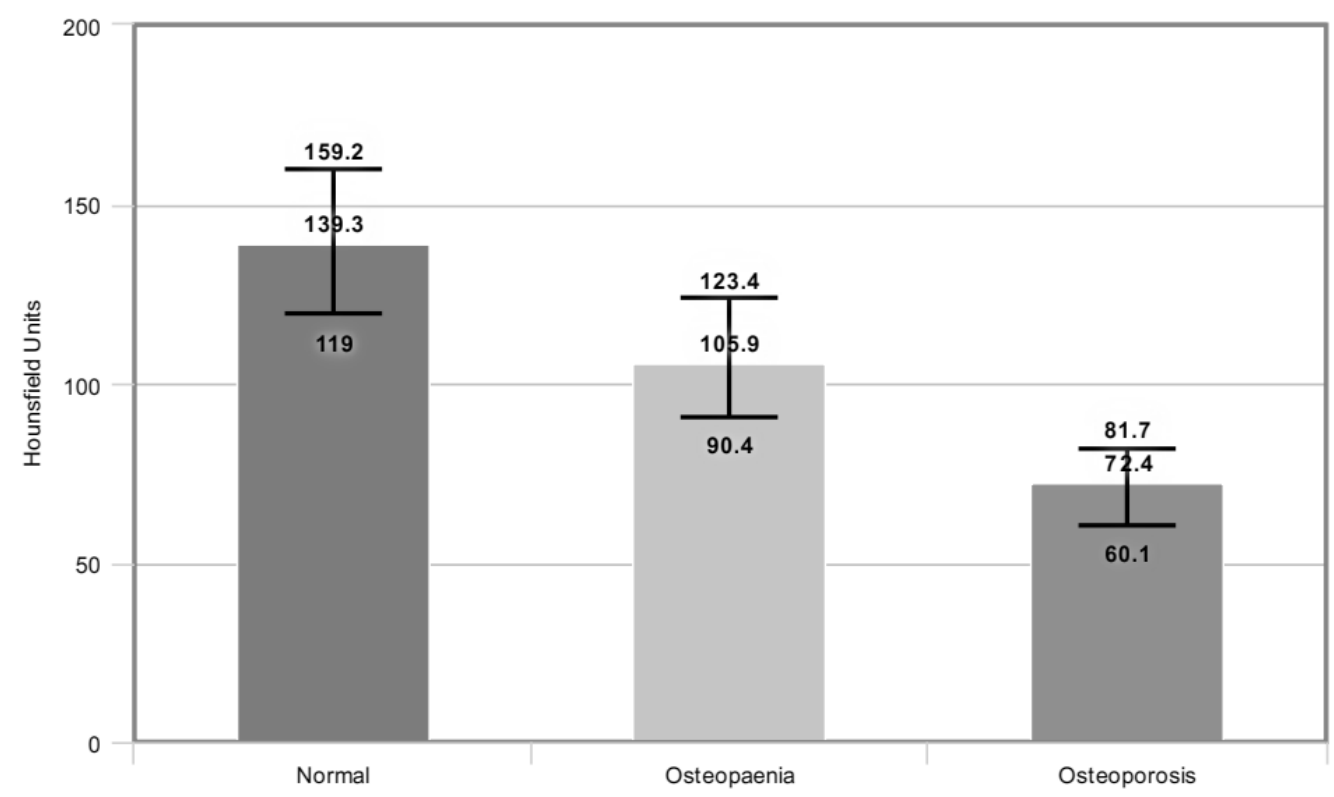

Figure 3: Mean $\mathrm{HU}$ values $( \pm \mathrm{SD})$ in the lumbar spine representative of normal bone, osteopenia, and osteoporosis. Error bars depict $95 \% \mathrm{Cl}$. 


\section{Conclusion}

In conclusion, this is the largest single study to date demonstrating that opportunistic lumbar spine CT scans can be used for osteoporosis screening without the need for additional imaging, radiation exposure, cost, or patient time. Furthermore, this information can be invaluable information for the surgeon preoperatively and enables appropriate treatments and referrals to be made. Further research is required with standardized methods and thresholds for this potentially highly impactful technique to be broadly applied.

\section{References}

1. Australian Bureau of Statistics (2018) Themes: Ageing, Australia.

2. Amarnath AL, Franks P, Robbins JA, Xing G, Fenton JJ (2015) Underuse and overuse of osteoporosis screening in a regional health system: A retrospective cohort study. J Gen Intern Med 30: 1733-1740.

3. Morris CA, Cabral D, Cheng H, Katz JN, Finkelstein JS, et al. (2004) Patterns of bone mineral density testing: current guidelines, testing rates, and interventions. J Gen Intern Med 19: 783-790.

4. Lewiecki EM, Gordon CM, Baim S, Leonard MB, Bishop NJ, et al. (2008) International Society for Clinical Densitometry 2007 Adult and Pediatric Official Positions. Bone 43: 1115-1121.

5. Bolotin HH (2007) DXA in vivo BMD methodology: An erroneous and misleading research and clinical gauge of bone mineral status, bone fragility, and bone remodelling. Bone 41: 138-154.

6. Yu EW, Thomas BJ, Brown JK, Finkelstein JS (2012) Simulated increases in body fat and errors in bone mineral density measurements by DXA and QCT. J Bone Miner Res 27: 119-124.

7. Gausden EB, Nwachukwu BU, Schreiber JJ, Lorich DG, Lane JM (2017) Opportunistic use of CT imaging for osteoporosis screening and bone density assessment: A qualitative systematic review. J Bone Joint Surg Am 99:15801590.

8. Genant HK, Boyd D (1977) Quantitative bone mineral analysis using dual energy computed tomography. Invest Radiol 12: 545-551.

9. Lee S, Chung CK, Oh SH, Park SB (2013) Correlation between bone minera density measured by dual-energy X-Ray absorptiometry and hounsfield units measured by diagnostic CT in lumbar spine. J Korean Neurosurg Soc 54: 384-389.
10. Goo HW (2012) CT radiation dose optimization and estimation: An update for radiologists. Korean J Radiol 13: 1-11.

11. Papadakis AE, Karantanas AH, Papadokostakis G, Petinellis E, Damilakis J (2009) Can abdominal multi-detector CT diagnose spinal osteoporosis? Eur Radiol 19: 172-176.

12. Schreiber JJ, Anderson PA, Hsu WK (2014) Use of computed tomography for assessing bone mineral density. Neurosurg Focus 37: E4.

13. Tay WL, Chui CK, Ong SH, Ng AC (2012) Osteoporosis screening using areal bone mineral density estimation from diagnostic CT images. Acad Radiol 19 1273-1282.

14. Link TM, Koppers BB, Licht T, Bauer J, Lu Y, et al. (2004) In vitro and in vivo spiral CT to determine bone mineral density: Initial experience in patients at risk for osteoporosis. Radiology 231: 805-811.

15. World Health Organization (2010) Prevention and management of osteoporosis. World Health Organiz Tech Rep Ser 921: 1-164.

16. Schreiber JJ, Anderson PA, Rosas HG, Buchholz AL, Au AG (2011) Hounsfield units for assessing bone mineral density and strength: a tool for osteoporosis management. J Bone Joint Surg Am 93: 1057-1063.

17. Pickhardt PJ, Pooler BD, Lauder T, Del Rio AM, Bruce RJ, et al. (2013) Opportunistic screening for osteoporosis using abdominal computed tomography scans obtained for other indications. Ann Intern Med 158: 588-595.

18. Grams AE, Rehwald R, Bartsch A, Honold S, Freyschlag CF, et al. (2016) Correlation between degenerative spine disease and bone marrow density: a retrospective investigation. BMC Med Imaging 16:17.

19. Rand T, Seidl G, Kainberger F, Resch A, Hittmair K, et al. (1997) Impact of spinal degenerative changes on the evaluation of bone mineral density with dual energy X-ray absorptiometry (DXA). Calcif Tissue Int 60: 430-433.

20. Paiva LC, Filardi S, Pinto-Neto AM, Samara A, Marques Neto JF (2002) Impact of degenerative radiographic abnormalities and vertebral fractures on spinal bone density of women with osteoporosis. Sao Paulo Med J 120: 9-12.

21. Raisz LG (2005) Clinical practice. Screening for osteoporosis. N Engl J Med 353: 164-171.

22. Schuit SC, Van der Klift M, Weel AE, De Laet CE, Burger H, et al. (2004) Fracture incidence and association with bone mineral density in elderly men and women: the Rotterdam Study. Bone 34: 195-202.

23. Yu W, Gluer CC, Fuerst T, Grampp S, Li J, et al. (1995) Influence of degenerative joint disease on spinal bone mineral measurements in postmenopausal women. Calcif Tissue Int 57: 169-174. 